\title{
Inonotus obliquus polysaccharide regulates gut microbiota of chronic pancreatitis in mice
}

\author{
Yang Hu${ }^{1,2,3}$, Chunying Teng ${ }^{1}$, Sumei Yu ${ }^{1}$, Xin Wang ${ }^{1}$, Jinsong Liang ${ }^{1}$, Xin Bai ${ }^{1}$, Liying Dong ${ }^{1}$, Tao Song ${ }^{1}$, Min Y ${ }^{4}$ \\ and Juanjuan $\mathrm{Qu}^{1 *}$
}

\begin{abstract}
Polysaccharide is efficient in attenuation of metabolic ailments and modulation of gut microbiota as prebiotics. The therapeutic effect of Inonotus obliquus polysaccharide (IOP) on chronic pancreatitis (CP) in mice has been validated in our previous study. However, it is not clear whether IOP is conducive to maintaining the homeostasis between gut microbiota and host. The aim of this study is to testify the potential effects of IOP on gut microbiota composition and diversity in mice with CP. The changes in glutathione peroxidase (GSH-P $)$, total antioxidant capacity (TAOC), tumor necrosis factor alpha (TNF-a), transforming growth factor beta (TGF- $\beta$ ), lipase and trypsin levels were measured by commercial assay kits, meanwhile the gut microbiota composition and diversity were analyzed by high throughput sequencing. The IOP treatment increased GSH-P and TAOC levels, and decreased TNF- $\alpha$, TGF- $\beta$, lipase and trypsin levels in CP mice. It was also observed that gut microbiota in IOP treated groups were less diverse than others in terms of lower Shannon diversity index and Chao 1 estimator. IOP increased the proportion of Bacteroidetes and decreased that of Firmicutes at phylum level. Bacteroidetes was found positively correlated with GSH-P and TAOC, and Firmicutes correlated with TNF-a, TGF- $\beta$, and lipase. In conclusion, administration of IOP could regulate gut microbiota composition and diversity to a healthy profile in mice with $\mathrm{CP}$, and some bacterial phylum significantly correlated with characteristic parameters.
\end{abstract}

Keywords: Inonotus obliquus polysaccharide, Chronic pancreatitis, Mice, Gut microbiota,

High throughput sequencing

\section{Introduction}

Chronic pancreatitis (CP) is a progressive and permanent destruction of the pancreas leading to insufficient exocrine and endocrine, and often chronic disabling pain. The complications of CP commonly include diabetes mellitus, cholangitis, ascites and even carcinoma of pancreas (Inui et al. 2013; Phillips 2012). In developed countries, $\mathrm{CP}$ incidence ranges from 3.5 to 10 per 100,000 populations, while the unhealthy lifestyle has caused a gradual rise in CP (Witt et al. 2007). Nowadays in China the incidence of $\mathrm{CP}$ is about to surpass $1 \%$ which is 20 times

\footnotetext{
*Correspondence: juanjuan4050234@163.com

${ }^{1}$ College of Resources and Environmental Science, Northeast Agricultural University, Harbin 150030, People's Republic of China

Full list of author information is available at the end of the article
}

higher than that in 1950s. The consumption of alcohol as well as genetic and environmental factor might cause CP (Braganza et al. 2011). Currently, oxidative stress has been implicated as a potential mechanism in etiology and pathology of CP (Zhou et al. 2015). 3, 5-Diethoxycarbonyl-1,4-dihydrocollidine (DDC) is a kind of SOD inhibitor that may cause oxidative damage and following fibrosis in pancreas (Matsumura et al. 2001). DDC has been managed to induce $\mathrm{CP}$ in mice (Matsumura et al. 2001; Fickert et al. 2014).

Under long-term oxidative stress, the invasion of pathogenic bacteria can destroy the microenvironment, disturbed the structure of intestinal flora, and ultimately lead to dysbiosis (Burcelin et al. 2011; Ley et al. 2008a, b). The correlation between the variation of gut microbiota and the development of bowel inflammatory, diverticulitis, 
diabetes, obesity etc. has been explicit, but it remains elusive as for CP (Rautava and Isolauri 2002; Berry et al. 2012; Daniels et al. 2014; He et al. 2015; Lim et al. 2015). Recently, Tan et al. reported the relationship between intestinal microbiota composition and inflammation involved in the progression of acute pancreatitis (Tan et al. 2015). Although the mechanism involved in these changes has not been fully elucidated, a major shift in gut microbiota composition was found in patients with severe and mild acute pancreatitis, especially an increasing relative abundance of Enterococcus and a decrease of Bifidobacterium.

Polysaccharides contribute to the proliferation of "good bacteria" and regulate microbial composition in host gut. Several polysaccharides like lentinan, glucans, mannans and xylans have the prebiotic effects on the intestines such as increasing the resistance of intestinal mucosa to inflammation and inhibiting the development of intestinal ulcers in rats (Singdevsachan et al. 2016). Shi et al. also found that a water-soluble-D-fructan extracted from the roots of Ophiopogon japonicas exhibited potent anti-obesity and hypoglycemic effects via regulating the gut microbiota of the host as prebiotics (Shi et al. 2015). Inonotus obliquus (also called Chaga), is a white-rot basidiomycete parasitizing on Betula (birch) trunks in low latitudes (about $45-50^{\circ} \mathrm{N}$ ) of Europe, Asia and North America. Experiments showed that I. obliquus could be used as drugs to prevent and cure cancer, diabetes, cerebrovascular diseases, etc. (Zhou et al. 2015; Ma et al. 2013). Inonotus obliquus polysaccharide (IOP) possesses comprehensive biological properties, especially antioxidant and anti-inflammatory activities. As a superior antioxidant, IOP has been used to attenuate CP in our previous research (Hu et al. 2016). However, the overall gut microbiota structure and diversity in CP mice administrated with IOP was ambiguous.

To verify the relationship between $\mathrm{CP}$ and gut microbiota, a clinical experiment in mice was conducted to evaluate the variations of glutathione peroxidase $\left(\mathrm{GSH}-\mathrm{P}_{\mathrm{X}}\right)$, total antioxidant capacity (TAOC), tumor necrosis factor alpha (TNF- $\alpha$ ), transforming growth factor beta (TGF- $\beta$ ), lipase, trypsin and the overall changes of gut microbiota in feces. The discovery of their correlation may be contributed to CP pathogenesis and therapy in terms of the intestinal ecosystem.

\section{Materials and methods}

\section{Chemicals and reagents}

Inonotus obliquus (CFCC83280) was provided by Harbin Baykaltai Bioengineering Co. LTD of China. Anionexchange DEAE cellulose column and Sephadex G-200 gel were obtained from Pharmacia (USA). Chloroform, butanol and ethanol were purchased from Kermel Chemical Reagent Co. (Tianjin, China). DDC was purchased from Sigma Chemical Co. (St Louis, USA). All solutions were prepared by analytical reagents and double distilled water. GSH- $\mathrm{P}_{\mathrm{X}}$, TAOC, TNF- $\alpha$, TGF- $\beta$, lipase and trypsin were detected by commercial assay kits purchased from the Nanjing Jiancheng Bioengineering Institute (Nanjing, China). DNA mini stool kit was purchased from Qiagen (Valencia, CA, USA). MetaVx ${ }^{\mathrm{TM}}$ library preparation kit was purchased from GENEWIZ Institute (South Plainfield, NJ, USA).

\section{Preparation of IOP}

IOP was prepared according to our previous procedure (Hu et al. 2016). Briefly, the dried sclerotia of I. obliquus were ground to powder and extracted with distilled water at $60{ }^{\circ} \mathrm{C}$ for $2.5 \mathrm{~h}$. The supernatant was concentrated and treated with Sevag reagent (Chloroform: butanol $=5: 1$ ) to remove protein. Then the supernatant was mixed with four volumes of $95 \%$ ethanol and kept at $4{ }^{\circ} \mathrm{C}$ for $12 \mathrm{~h}$. Crude IOP was centrifuged, lyophilized and further purified in an anion-exchange DEAE cellulose column $(50 \mathrm{~cm} \times 2.6 \mathrm{~cm})$ which was eluted with $0.05 \mathrm{M}, 0.1 \mathrm{M}$ and $0.2 \mathrm{M} \mathrm{NaCl}$ solution and a Sephadex G-200 gel column $(1.6 \mathrm{~cm} \times 40 \mathrm{~cm})$. The obtained IOP is a homogeneous polysaccharide with molecular weight of $32.5 \mathrm{kDa}$, polysaccharide content of $98.6 \%$, and monosaccharide composition of Man, Rha, Glu, Gal, Xyl and Ara in a molar ratio of 9.8:13.6:29.1:20.5:21.6:5.4 (Hu et al. 2016).

\section{Toxicity test}

Toxicity test was performed according to our previous study (Hu et al. 2017). Briefly, specific pathogen-free male ICR mice (18-22 g) were purchased from Drug Safety Evaluation Center of Heilongjiang University of Chinese Medicine. IOP was administrated to mice at a dose of $1 \mathrm{~g} / \mathrm{kg}$ body weight by oral gavage three times a day. Control group received saline solution. Sterilized water and standard chow were provided for all mice. The mortality and side effects of mice were observed for $72 \mathrm{~h}$.

\section{Experimental design}

Mice were randomly divided into six groups with ten mice in each group: three IOP treated groups (IOP-L, IOP-M and IOP-H), Qingyilidan granule treated group (PC), model control group (MC), and normal control group (NC). Except for mice in NC group, all mice were received intraperitoneal injections with DDC $(10 \%, 0.5 \mathrm{~g} /$ $\mathrm{kg}$ body weight) twice a week for continuous four weeks (Fickert et al. 2014). Mice in IOP treated groups were orally administrated with IOP at a dosage of 0.1 (IOPL), 0.2 (IOP-M) and $0.4 \mathrm{~g} / \mathrm{kg} /$ day (IOP-H) body weight for continuous four weeks from the second week of the DDC injection, respectively. PC group was daily fed with Qingyilidan granule at a dose of $3.7 \mathrm{~g} / \mathrm{kg} /$ day. Mice in $\mathrm{MC}$ 
and $\mathrm{NC}$ groups were given normal saline to osmotic pressure of $0.9 \%$. All mice were kept in a $12 \mathrm{~h}$ dark/light cycle room with humidity-control at a constant temperature of $25{ }^{\circ} \mathrm{C}$. They all had free access to sterilized water and standard chow. After 5-week experiment, all mice were scarified via cervical dislocation.

\section{Measurement of GSH-Px, TAOC, TNF- $\alpha$, TGF- $\beta$, lipase and trypsin}

The pancreas tissue was homogenized with normal saline by sonication. The activities of GSH-Px and TAOC were determined using commercial kits, and they were expressed as $\mathrm{U} / \mathrm{mg}$ wet weight of pancreatic tissue.

Blood samples were collected from the eye vein by removing eyeball. Then serum was separated by centrifugation at $3000 \mathrm{rpm}$ for $10 \mathrm{~min}$ at $4{ }^{\circ} \mathrm{C}$ and stored at $-80{ }^{\circ} \mathrm{C}$ until use. The contents of serum TNF- $\alpha$ and TGF- $\beta$ were determined by commercial assay kits according to manufacturer's instructions. The activities of TNF- $\alpha$ and TGF- $\beta$ were expressed as $\mathrm{pg} / \mathrm{mL}$.

Serum lipase and pancreatic trypsin were quantified using detection kits according to manufacturer's instructions. Lipase and trypsin contents were expressed as U/L and $\mathrm{pmol} / \mathrm{mg}$ respectively. Each sample was analyzed in triplicate.

\section{Preparation of genomic DNA from fecal samples}

The fecal samples were collected from each group at the end of the experiment. Total DNA was extracted according to the method of $\mathrm{Yu}$ and Morrison ( $\mathrm{Yu}$ and Morrison 2004). $0.5 \mathrm{~g}$ (wet weight) of fecal sample was suspended in $2 \mathrm{~mL}$ of breaking buffer $(0.5 \mathrm{M} \mathrm{NaCl}, 50 \mathrm{mM}$ Tris$\mathrm{HCl}, 50 \mathrm{mM}$ EDTA, $4 \%$ sodium dodecyl sulfate) at $70{ }^{\circ} \mathrm{C}$ for $15 \mathrm{~min}$ after shaking for $30 \mathrm{~s}$. The mixture was centrifuged at $6000 \mathrm{rpm}$ for $5 \mathrm{~min}$ at $4{ }^{\circ} \mathrm{C}$ and the supernatant was removed and retained. This procedure was repeated for 3 times and the obtained supernatants were pooled. Nucleic acids were extracted sequentially with ammonium acetate and isopropanol. They were then treated with DNase-free RNase, proteinase K, and further purified with DNA mini stool kit.

\section{MetaVx ${ }^{\mathrm{TM}}$ library preparation and Illumina MiSeq sequencing}

Next generation sequencing library preparations and Illumina MiSeq sequencing were conducted at GENEWIZ, Inc. (Beijing, China). DNA samples were quantified using a Qubit2.0 Fluorometer (Invitrogen, Carlsbad, CA) and DNA quality was checked on a $0.8 \%$ agarose gel. $5-50 \mathrm{ng}$ DNA was used to generate amplicons using a MetaVx ${ }^{\mathrm{TM}}$ library preparation kit. A panel of proprietary primers was designed to anneal to the relatively conserved regions bordering V3, V4, and V5 hypervariable regions. The V3 and $\mathrm{V} 4$ regions were amplified using forward primer in sequence of CCTACGGRRBGCASCAGKVRVGAAT and reverse primer in sequence of GGACTACNVGGGTWTCTAATCC. The V4 and V5 regions were amplified using forward primer in sequence of GTGYCAGCMGCCGCGGTAA and reverse primer in sequence of CTTGTGCGGKCCCCCGYCAATTC. All PCR products were purified with the QIAgen DNA Mini Stool Kit. DNA libraries were validated using an Agilent 2100 Bioanalyzer (Agilent Technologies, Palo Alto, CA, USA), and quantified by Qubit and real time PCR (Applied Biosystems, Carlsbad, CA, USA). DNA libraries were multiplexed and loaded on an Illumina MiSeq instrument according to manufacturer's instructions (Illumina, San Diego, CA, USA) by GENEWIZ.

\section{Bioinformatic and statistical analysis}

The sequences were clustered into operational taxonomic units (OTUs) using a 97\% identity cut-off. OTUs were used for diversity and richness analysis. Partial least square discriminate analysis (PLS-DA) was used to identify OTUs by Simca-P+ software (version 12.0, Umetrics AB, Umea, Sweden). Variable importance in projection (VIP) was used to select the key OTUs based on their contribution to the biochemical characteristics of our study. The OTUs with the highest contribution (VIP score $>1.0$ ) were translated to the key OTUs and used to clarify the relationships between groups and key OTUs. Pearson correlation analysis in SPSS 18.0 (SPSS Inc., Chicago, IL, USA) was used to evaluate the correlations between biochemical characteristics and gut microbiota at phylum level. Clustering analysis and heatmap were performed using Mothur and R software (http://www.mothur.org/wiki/MainPage). Canonical correspondence analysis (CCA) was determined by Canoco 4.5 (Biometrics, Wageningen, The Netherlands). Sequences used in this study were deposited to the NCBI Sequence Read Archive (accession number SRP067729).

Biochemical characteristics data were analyzed with SPSS 18.0. General characteristics were expressed as median and mean or percentages. Statistical analyses were carried out using one-way analysis of variance. Statistical significance was calculated by Student's t test and a probability value $P<0.05$ or $P<0.01$ was considered to be significant in statistic.

\section{Results}

Toxicity test

During the experimental period, no death was observed. The mice treated with IOP did not show any side effects. 


\section{Biochemical characteristics analysis of GSH-Px, TAOC, TNF- $\alpha$, TGF- $\beta$, lipase and trypsin}

As an important enzyme in superoxide degradation, GSH-Px has been taken as an index to the antioxidative status in organism (Rotruck et al. 1973), and a decrease in GSH-Px level is generally observed in carcinoma, sclerosis and CP patients (Sehitogullari et al. 2014; AdamczykSowa et al. 2012; Girish et al. 2011). The level of TAOC also can reflect the capacity of nonenzymatic antioxidant defense system (Li et al. 2007). As shown in Fig. 1A, B, the pancreatic GSH-Px and TAOC in MC group were lowest indicating that DDC inducement had caused pancreatitis in mice. The higher levels of GSH-Px and TAOC in all IOP groups than those in MC group $(P<0.01)$ illustrated that administration of IOP could prevent pancreas from oxidative damage. Moreover, IOP treatment on GSH-Px and TAOC presented a dose-dependently effect, the GSH-Px level of IOP-L, IOP-M and IOP-H group was $32.3 \pm 0.8,45.1 \pm 0.7$ and $45.9 \pm 0.8 \mathrm{U} / \mathrm{mg}$ respectively, the TAOC level was $0.96 \pm 0.04,1.21 \pm 0.06$, and $1.54 \pm 0.02 \mathrm{U} / \mathrm{mg}$ respectively. And the level of TAOC in IOP-H group was insignificantly different from that in NC group $(P>0.05)$.

Levels of serum TNF- $\alpha$ and TGF- $\beta$ in mice at the end of experiment were given in Fig. 1C, D. The value of TNF- $\alpha$ in $\mathrm{MC}$ group was $53.9 \pm 0.7 \mathrm{pg} / \mathrm{mL}$ which was about 2.5 times higher than that in NC group $(21.8 \pm 0.9 \mathrm{pg} / \mathrm{mL})$ $(P<0.01)$, indicating that the prolonged inflammatory response in $\mathrm{CP}$ mice might result in an increase in the systemic concentration of TNF- $\alpha$ (Petersen and Pedersen 2005). In IOP-L, IOP-M, IOP-H and Qingyilidan granule group, TNF- $\alpha$ level in mice was about 22.9, 14.3, 14.1 and $15.8 \mathrm{pg} / \mathrm{mL}$ higher than that in NC group respectively, but greatly lower than that in MC group $(P<0.01)$. Likewise, the level of TGF- $\beta$ in MC group increased $383.04 \mathrm{pg} / \mathrm{mL}$ compared with NC group $(P<0.01)$, indicating that DDC inducement had caused the injury of pancreas in mice (Schneider et al. 2004). However, the levels of TGF- $\beta$ were significantly decreased in the IOP and $\mathrm{PC}$ groups when compared with $\mathrm{MC}$ group.

Data on serum lipase and pancreatic trypsin levels in mice at the end of the experiment were presented in Fig. 1E, F. The highest level of lipase was found in MC group which was 1.2, 1.4, 1.4, 1.4 and 1.8 times higher than that in IOP-L, IOP-M, IOP-H, PC and NC group respectively. Benini et al. found that an increase in lipase level was always detected in patients with CP (Benini et al. 1987). The curative effect of IOP was not invariably dose-dependent, just as the high and moderate dose of IOP had a same effect on lipase $(P>0.05)$. Similarly, activity of trypsin in $\mathrm{MC}$ group was higher than that in NC group. However, IOP and Qingyilidan granule decreased the level of trypsin and the insignificant difference between PC and IOP groups $(P>0.05)$ suggested that activity of IOP amounted to the commercial herb medicine that is generally used for CP therapy.

\section{OTU, cluster analysis, diversity and richness of six groups}

For six groups, variable regions (V3-V5) of the bacterial $16 \mathrm{~S}$ rRNA gene were amplified by PCR. From all the fecal samples, a dataset consisting of 409,812 high-quality $16 \mathrm{~S}$ rRNA gene sequences was obtained (Table 1). A total of 47,950 OTUs were identified based on the conventional criterion of $97 \%$ similarity (equal to species level), more specifically, 9652 OTUs for IOP-L group, 9439 OTUs for IOP-M group, 5918 OTUs for IOP-H group, and 6903 OTUs for PC group, 10815 OTUs for MC group, 5223 OTUs for NC group with an average sequence length of 298 bp.

Cluster analysis using unweighted pair group method with arithmetic mean (UPGMA) was shown in Fig. 2. Based on cluster analysis for gut microbiota structure, an obvious difference was shown among the six groups which were consistent with the divergence of biochemical characteristics. Genetic distance of cluster analysis showed that the six groups could be divided into two branches. IOP-H, IOP-M, IOP-L, PC and NC groups clustered in one branch with IOP-H group on the first grade. MC group was categorized as an independent branch, indicating a low similarity with others.

Shannon diversity index and Chao 1 estimator were used to describe the diversity and richness of microbiota. In this study, gut microbiota diversity and richness were significantly increased by DDC inducement as presented in MC group (Table 1). For three IOP groups, Shannon diversity indexes were lower than that of $\mathrm{MC}$, but higher than that of NC. Chao 1 estimator of $\mathrm{NC}$ group was 20227.70, but that of MC group reached 57,089.69. The results indicated the ecological balance of gut microbiota was disrupted in CP mice. However, IOP and Qingyilidan granule treatments were conducive to maintaining the microbiota structure and richness to normal level as that in $\mathrm{NC}$ group. And with the increasing dose of IOP, the community richness was correspondingly declined.

\section{Correlation analysis}

Principal coordinates analysis (PCoA) is usually used for visualizing and analyzing the association of sample metadata, it can also reveal the divergency of bacterial community structure. The PCoA plots of six groups were shown in Fig. 3. Figure 3a displayed that all IOP, PC and NC groups assembled in the right area of the bottom, MC group scattered in another area. It indicated that gut microbiota in three IOP treated groups and PC group were relevant to that in $\mathrm{NC}$ group, but $\mathrm{MC}$ group far from other groups with PC1 accounting for 33.99\% 

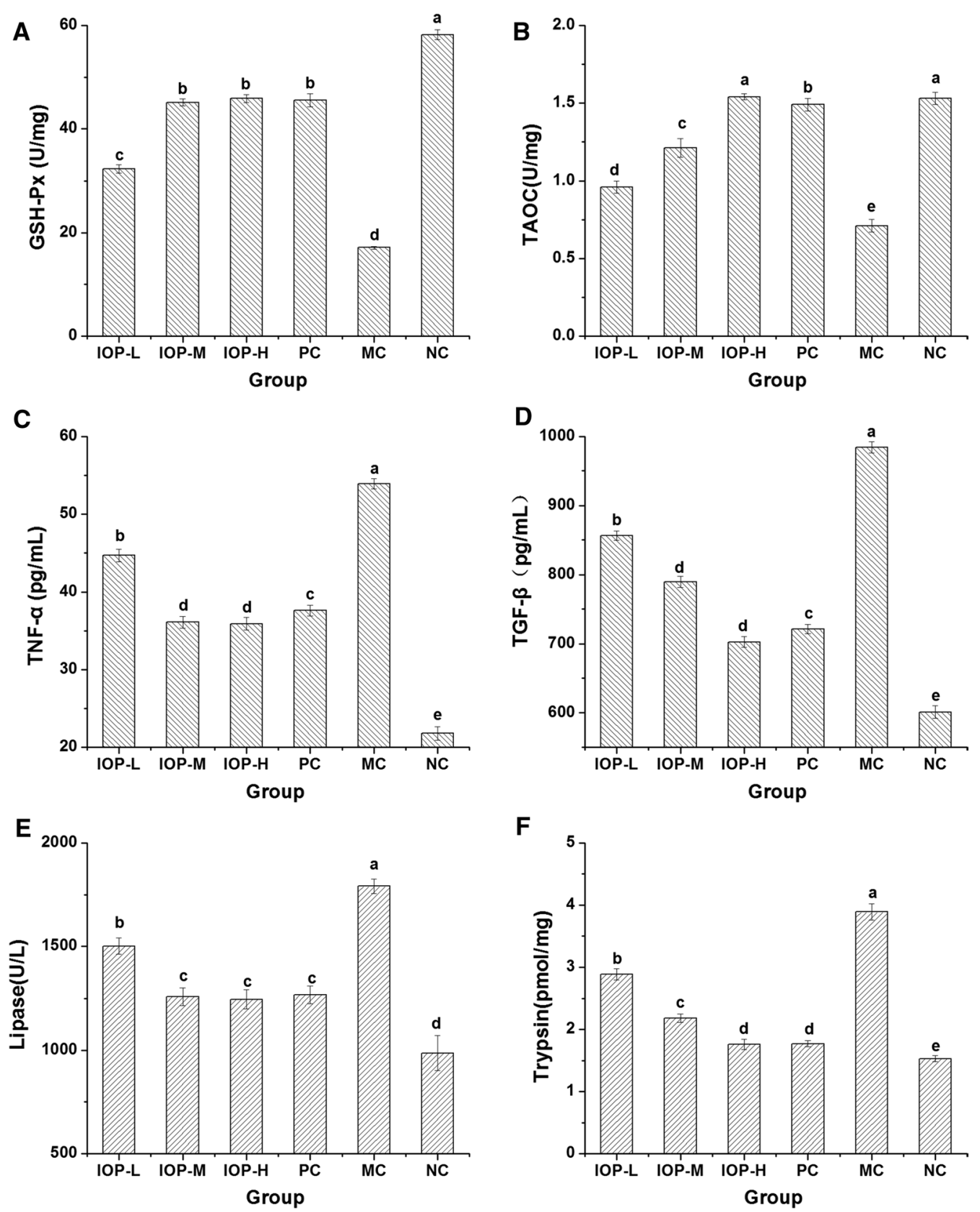

Fig. 1 Effect of IOP on biochemical parameters in CP mice induced by DDC. A Effect on pancreas GSH-Px level. B Effect on pancreas TAOC level. C Effect on serum TNF-a level. D Effect on serum TGF- $\beta$ level. E Effect on serum lipase level. $\mathbf{F}$ Effect on pancreas trypsin level. Values are shown as the mean $\pm S D(n=10)$. Means within each error bar having different letters are significantly different $(p<0.05)$

of the total variation (Fig. 3a). The gut microbiota in six groups also differed from each other along PC2 and PC3 (Fig. 3b). Three IOP groups were far from MC group along $\mathrm{PC} 2$ and $\mathrm{PC} 3$, and they were distinctively apart from NC group. Overall, IOP and PC groups were far from MC group, but near to the NC group, and the PCoA plot visualization explained over $83 \%$ of the microbiota variation. The results showed that the variation of gut microbiota structure was closely related to the degree of $\mathrm{CP}$, and IOP could remarkably change the structure of gut microbiota.

61 Key OTUs were selected by PLS-DA with highest contribution to the biochemical characteristics (VIP score $>1.0$ ). The heatmap shown in Fig. 4 a indicated the 
Table 1 Illumina MiSeq sequencing data

\begin{tabular}{lrrll}
\hline & Seq_num & OTU & $\begin{array}{l}\text { Shannon } \\
\text { diversity index }\end{array}$ & $\begin{array}{l}\text { Chao 1 } \\
\text { estimator }\end{array}$ \\
\hline IOP-L & 80,150 & 9652 & 8.71 & $45,078.72$ \\
IOP-M & 75,410 & 9439 & 8.69 & $39,521.43$ \\
IOP-H & 53,515 & 5918 & 8.44 & $23,794.53$ \\
PC & 66,853 & 6903 & 8.50 & $33,496.84$ \\
MC & 90,192 & 10,815 & 9.00 & $57,089.69$ \\
NC & 43,692 & 5223 & 8.24 & $20,227.70$ \\
\hline
\end{tabular}

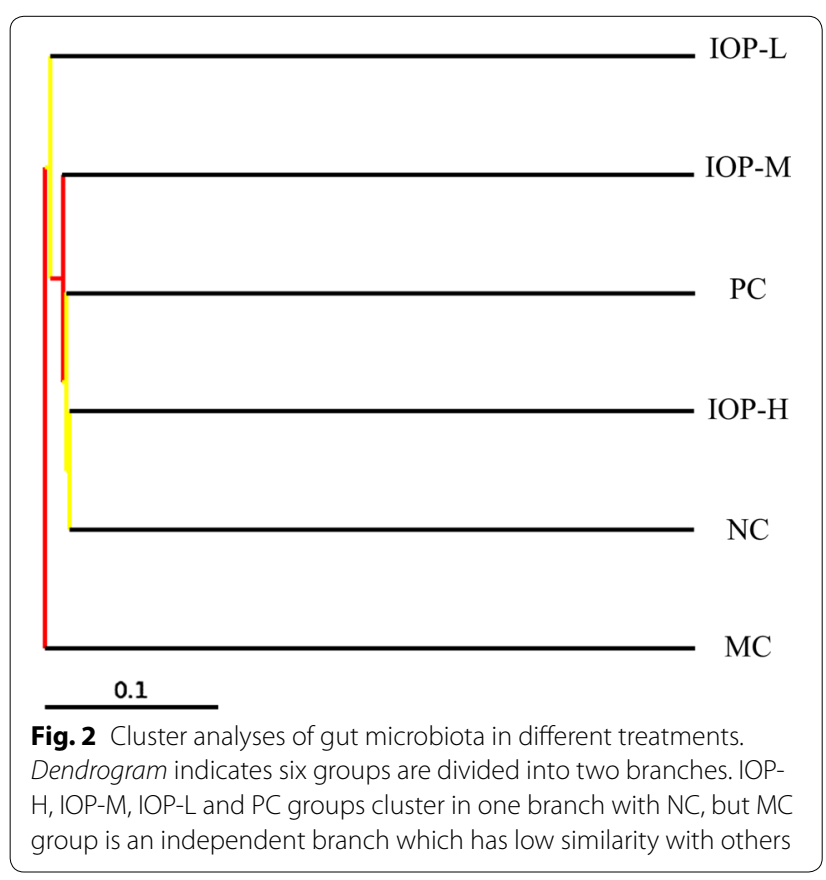

relative percentage of key OTUs in the six groups. The relative value was presented by the color intensity and the legend was illustrated in the figure. The structure of gut microbiota in IOP, PC and NC groups shared higher similarity compared with that in MC group.

Based on Pearson correlation analysis, the correlations of six biochemical parameters and gut microbiota at phylum level were shown in Fig. 4b. This plot revealed that Bacteroidetes (increased by IOP) had significant positive correlation with GSH-Px $(\mathrm{P}<0.05)$ and TAOC $(\mathrm{P}<0.05)$, while negative correlation with TNF- $\alpha(\mathrm{P}<0.01)$, TGF- $\beta$ $(\mathrm{P}<0.01)$, lipase $(\mathrm{P}<0.05)$ and trypsin $(\mathrm{P}<0.05)$. Firmicutes (decreased by IOP) was negatively associated with GSH-Px $(\mathrm{P}<0.05)$, but positively associated with TNF- $\alpha$ $(\mathrm{P}<0.05)$, TGF- $\beta(\mathrm{P}<0.05)$, and lipase $(\mathrm{P}<0.05)$.

Canonical correspondence analysis (CCA) of six groups was shown in Fig. 4c. 58.79\% of the variations were explained by CCA1 while $25.12 \%$ were explained by CCA2. The biplot showed that gut microbiota was significantly shaped by GSH-Px, TAOC, TNF- $\alpha$, TGF- $\beta$, lipase and trypsin. The results indicated that biochemical parameters and their interactions might be essential to the maintenance of gut microbiota structure. PC and IOP-H group was close to each other and they were not far from NC group. This result was in accordance with that of PCoA analysis.

\section{Variation of gut microbiota composition at phylum and genus level}

In order to explore the overall variation in gut microbiota composition among six groups, the community difference at phylum and genus level was compared. Based on the core OTUs of taxonomic database analyzed by program QIIME, a total of 7 major phyla were identified in the six groups (Fig. 5a). The predominant phylum was Bacteroidetes, which contributed $65.05 \%$ to the total sequence reads in NC group and $47.47 \%$ in $\mathrm{MC}$ group. However, the proportion of Bacteroidetes was increased about 4.55, 9.56, 17.48 and $20.81 \%$ in IOP-L, IOP-M, IOP-H and PC groups respectively, compared with that in $\mathrm{MC}$ group. Firmicutes was the subdominant phylum, which contributed $47.52 \%$ to the total sequence reads in MC group. Proportions of Firmicutes in IOP and Qingyilidan groups were all significantly lower than those in $\mathrm{MC}$ group, but higher than that in NC group. And the relative abundance of Firmicutes was decreased 14.46 and $15.57 \%$ in IOP-H and PC group compared with that in MC group. Other phyla including Actinobacteria, Candidate division TM7, Cyanobacteria, Proteobacteria and Tenericutes, together contributed a lower percentage to the total bacterial sequences. Obviously, the discrepancy at phylum level suggested the difference in gut microbiota composition among six groups (Fig. 5b).

At genus level, the entire sequences of the six groups could be assigned to fourteen major genera. Lactobacillus was the most predominant genus in six groups, accounting for $13.00 \%$ of the total bacterial sequences. The subdominant genus Bacteroides and the following twelve genera were shown in Fig. 5c. DDC inducement decreased the proportion of Lactobacillus, Bacteroides, unclassified S24-7, unclassified Lachnospiraceae, unclassified Prevotellaceae, Roseburia and Prevotella, but increased the proportion of Alistipes, Incertae_Sedis, Helicobacter, Parabacteroides and Rikenella in MC group compared with that in NC group (Fig. 5d). The changes of unclassified Ruminococcaceae and RC9_gut_group were not obvious. While the results showed that both IOP and Qingyilidan granule had remarkable effects on gut microbiota composition. The proportions of Lactobacillus, Bacteroides, unclassified S24-7, unclassified Lachnospiraceae, unclassified Prevotellaceae, Roseburia and Prevotella were greatly increased in IOP or PC groups 

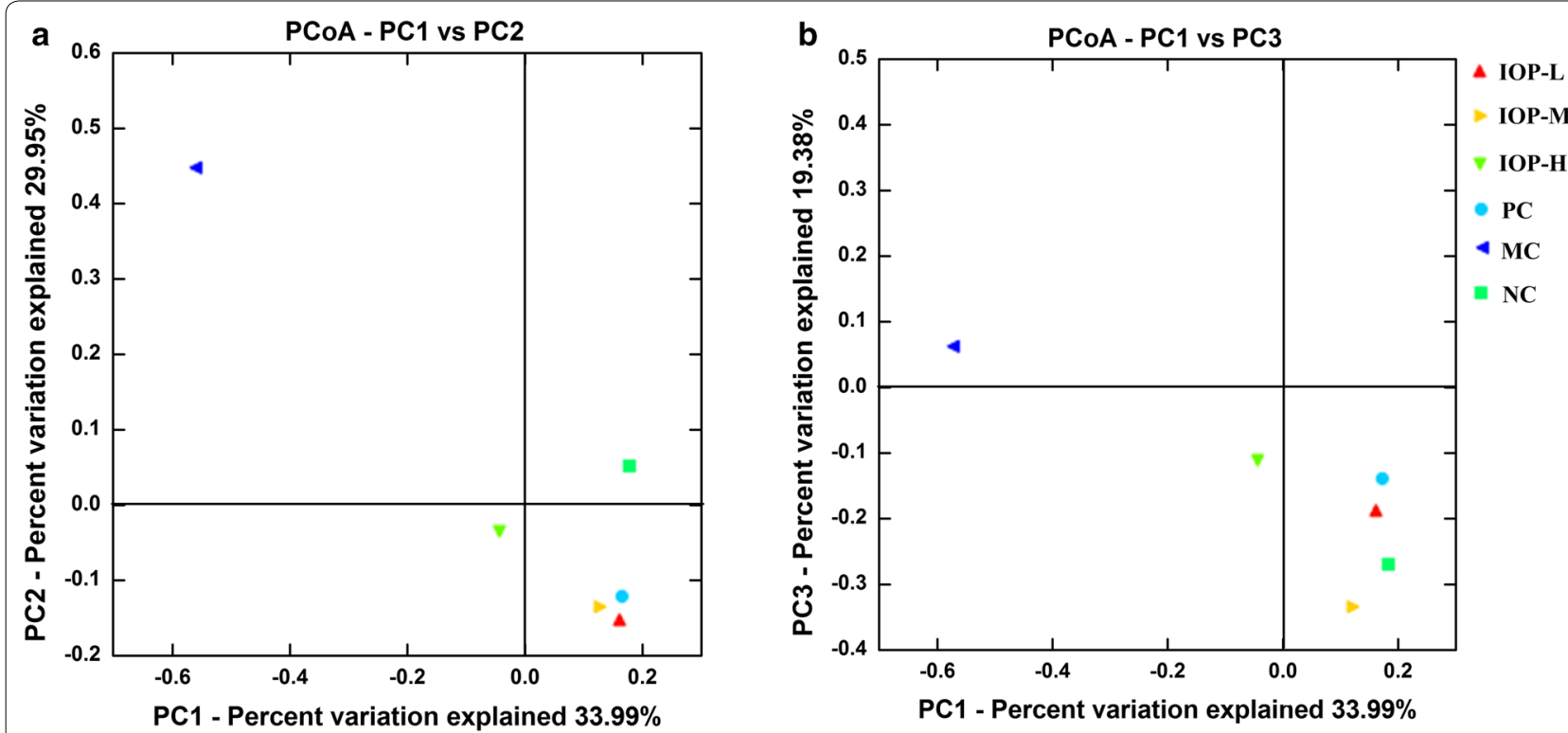

Fig. 3 Unweighted principal coordinate analysis plots: Unweighted UniFrac PCoA plotted against PC1 versus PC2 axes (a) and PC1 versus PC3 axes (b). The plots show the clustering pattern among IOP-L, IOP-M, IOP-H, PC, MC and NC groups. MC group is far from the other five groups, and the plots indicate the change of clustering after DDC injection and IOP intake
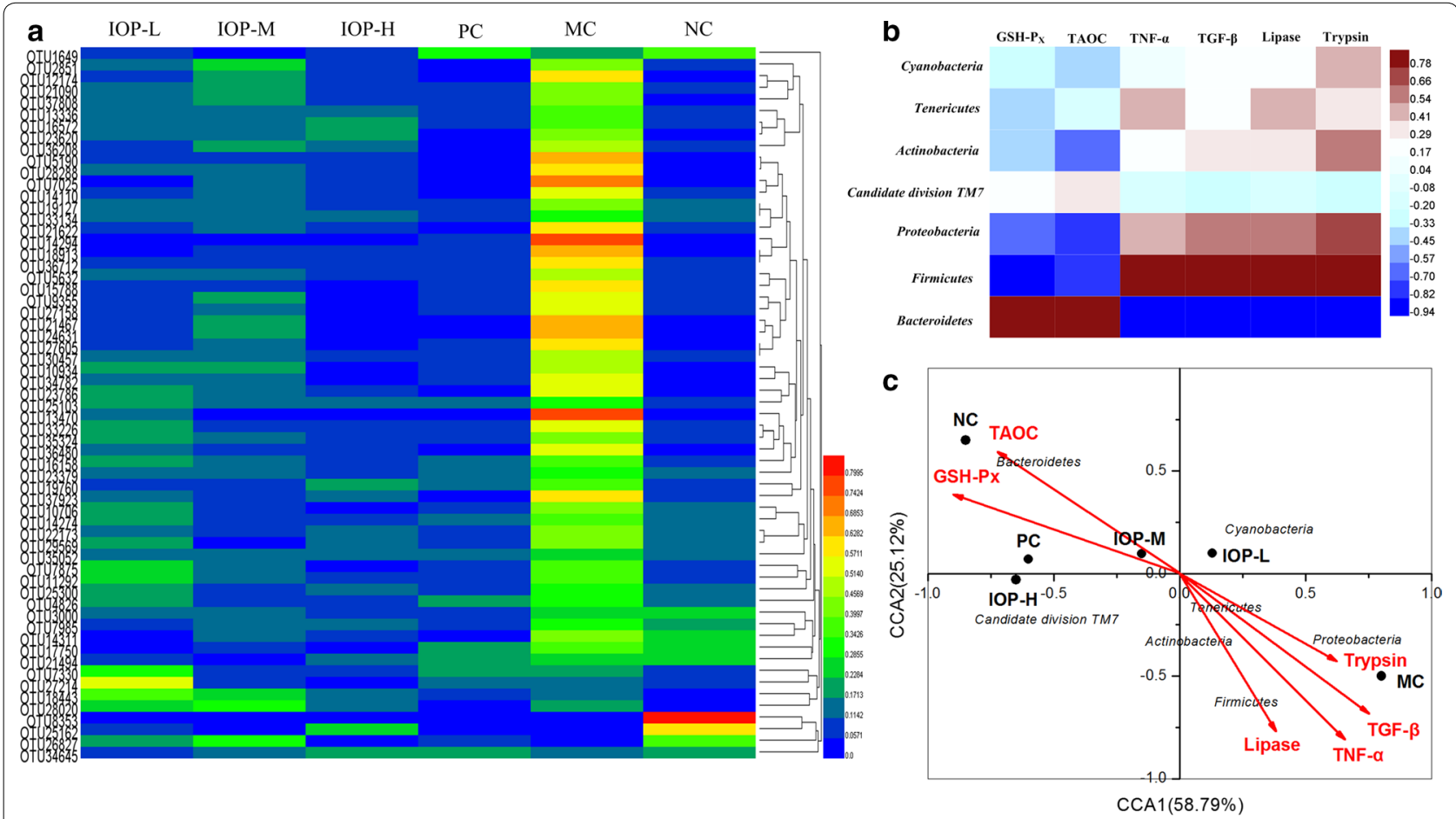

Fig. 4 Correlation analysis. a Heatmap of key OTUs in the six groups. b Heatmap of biochemical parameters and gut microbiota at phylum level. c Canonical correspondence analysis (CCA) of six groups 

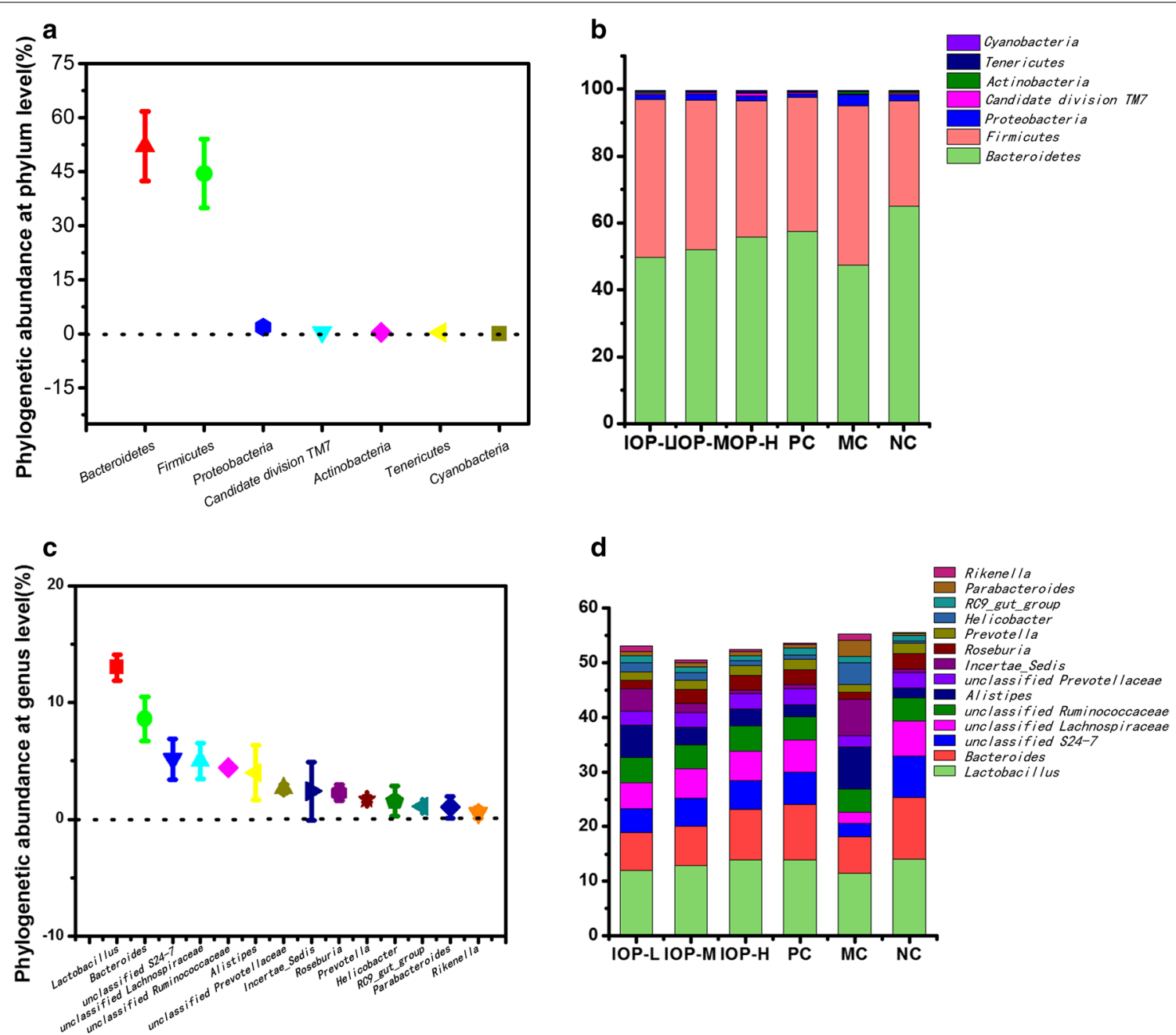

Fig. 5 Relative abundance of gut microbiota at phylum and genus level. a Phylogenetic abundance at phylum level. b Dominant phyla in each group. c Phylogenetic abundance at genus level. d Dominant genera in each group

compared with those in MC group, but those of Alistipes, Incertae_Sedis, Helicobacter, Parabacteroides and Rikenella were decreased.

\section{Discussion}

A disturbed microbiota rather than a single organism is supposed to be the pathologic agent for some chronic diseases, represented by an increase in bacterial diversity and/or an overgrowth in aggressive bacteria (Daniels et al. 2014; Walker et al. 2011). The variability of gut microbiota is possibly related to systemic inflammation. Daniels et al. found a higher diversity in diverticulitis for Proteobacteria, which also led to an alteration in diversity of other phyla together (Daniels et al. 2014). Administration of DDC stimulates the production of reactive oxygen species (ROS) which triggers the mitogen-activated protein kinase (MAPK)/Nuclear factor-kappa B (NF-kB) pathway and results in the production of proinflammatory cytokines, subsequently creates a hostile environment adjacent to the mucosal surface and cause a variation in gut microbiota (Silva et al. 2010; Siriwardena 2014). Although endogenous antioxidant system can prevent the toxic effect of ROS, however, excessive ROS generation caused by DDC may overwhelm the natural antioxidant defense system. Studies in mice with colitis showed that quantitative growth of Verrucomicrobia, Proteobacteria and specifically Bacteroidetes were almost linearly decreased as weight loss progressed (Vanhooren et al. 2013). While research also revealed that epithelium devoted to clear microbes away from mucosal surface in mounting responses and improve the proliferation of intestinal probiotics. But DDC inducement destroyed 
mucosal surface, which was detrimental to maintaining the stability of gut microbiota, and down-regulating the proliferation of intestinal probiotics.

In our study, IOP greatly decreased the Seq_num and OTU due to the loss of gut microbiota, which presumably provided space for normal bacteria to colonize in the gut, and a scenario also has been observed in gut microbiota variation of malnourished children in Bangladesh (Monira et al. 2011). Shannon diversity index and Chao 1 estimator showed that $\mathrm{CP}$ would increase the diversity and richness of mice, but IOP would decrease it. These were consistent with the study of $\mathrm{Xu}$ et al. that Lentinula edodes-derived polysaccharide can reduce the richness, diversity and evenness of microbial communities in cecum and colon (Xu et al. 2015).

In correlation analysis of the variation of gut microbiota and chemical parameters, significant correlations were found in an increase of Bacteroidetes and a decrease of Firmicutes, Protecbacteria, Actinobacteria and Cyanobacteria. The relative abundance of two dominant phyla, the Bacteroidetes and the Firmicutes, appears to play a role in the ability of the microbiome to harvest energy from the diet (Turnbaugh et al. 2006; Ley et al. 2005). Polsaccharides are hard to digest by human being, but recent researches have demonstrated that some microbes in the human gut can produce enzymes to hydrolyze complex polysaccharides to easily adsorbed monosaccharides (Bolam and Sonnenburg 2011). Bacteria in Bacteroidetes phylum may produce different kinds of polysaccharidedegrading enzymes, while Firmicutes phylum contains fewer microbes with the capacity of polysaccharides degradation (Bolam and Sonnenburg 2011; Ravcheev et al. 2013). Similar to other studies that Bacteroidetes and Firmicutes are predominant phyla in the gut flora (Ley et al. 2008a, b). As a dietary fungal polysaccharide, IOP has many beneficial effects on the host's health. Oral administration of IOP may drive qualitative and selective changes in the composition of the gut microbiota (Wang et al. 2014; Wu et al. 2011). When CP mice were administrated with IOP, the proportion of Bacteroidetes was increased with the increasing dose of IOP, but Firrmicutes was decreased.

Some genera such as Bacteroides, Prevotella and Lactobacillus in gut microbiota play an important part in the hydrolyzation of polysaccharides into short chain fatty acids (SCFA) (Bach-Knudsen et al. 2012). Li et al. found that Bacteroides could produce high levels of SCFA by fermentation of indigestible plant-derived substrates in fish intestine ( $\mathrm{Li}$ et al. 2015). Li et al. also found that Prevotella contained highly active hemicellulolytic and proteolytic enzymes, which could degrade polysaccharide, xylan and starch ( $\mathrm{Li}$ et al. 2014). In the study of Reilly, oat polysaccharide increased the amount of
Lactobacilli in intestinal microbiota and SCFA concentrations in pigs (Reilly 2010). The production of SCFA can provide energy for microbes, modulating immune responses and maintaining the epithelial barrier function (Brown et al. 2011). In our study, Bacteroides, Prevotella and Lactobacillus were richest in NC group but rarest in $\mathrm{MC}$ group, among all treatment groups, these three genera were more abundant in IOP-H group. This was consistent with the findings of Maslowski et al. that low concentrations of SCFA in germ-free mice would exacerbate inflammatory responses (Maslowski et al. 2009). An early study suggests that dietary fiber fed to conventional mice is capable to stimulate intestinal epithelial cell proliferation (Goodlad et al. 1989). In our study, IOP may also keep the integrity of epithelium, inhibit the proliferation of Alistipes, Incertae_Sedis, Helicobacter, Parabacteroides and Rikenella and alter the diversity of the intestinal probiotics in a dose-dependent way. However, a large number of unclassified and uncultured genera were also shown by sequence databases, the relations between $\mathrm{CP}$ and gut microbiota composition and how gut microbiota utilize IOP under oxidant injury need further research.

In summary, our results showed that IOP exerted pharmacological influence on $\mathrm{CP}$ related parameters in terms of increasing GSH-P $\mathrm{P}_{\mathrm{X}}$ and TAOC level and decreasing TNF- $\alpha$, TGF- $\beta$, lipase and trypsin levels. Meanwhile, IOP reduced the gut microbiota diversity and richness, decreased the relative abundance of Firmicutes, increased the Bacteroidetes at phylum level, and regulated gut microbiota toward a healthy profile at genus level. Moreover, the variations in gut microbiota were correlated with biochemistry parameters. The favorable biochemical characteristics and gut microbiota suggest that IOP has strong activity for ameliorating $\mathrm{CP}$ and a beneficial effect on gut microbiota.

\section{Abbreviations \\ CP: chronic pancreatits; DDC: 3,5-diethoxycarbonyl-1,4-dihydrocollidine; IOP. Inonotus obliquus polysaccharide; GSH-P : glutathione peroxidase; TAOC: total antioxidant capacity; TNF-a: tumor necrosis factor alpha; TGF- $\beta$ : transforming growth factor beta; IOP-L: IOP treated group at the dosage of $0.1 \mathrm{~g} / \mathrm{kg} /$ day IOP-M: IOP treated group at the dosage of $0.2 \mathrm{~g} / \mathrm{kg} /$ day; IOP-H: IOP treated group at the dosage of $0.4 \mathrm{~g} / \mathrm{kg} /$ day; $\mathrm{PC}$ : qingyilidan granule treated group; MC: model control group; NC: normal control group; OTUs: operational taxonomic units; PLS-DA: partial least square discriminate analysis; VIP: variable importance in projection; CCA: canonical correspondence analysis; UPGMA: unweighted pair group method with arithmetic mean; PCoA: principal coordi- nates analysis; ROS: reactive oxygen species; MAPK: mitogen-activated protein kinase; NF-kB: nuclear factor-kappa B; SCFA: short chain fatty acids.}

\section{Authors' contributions}

YH and JQ designed the experiments. YH and MY carried out all the experiments. YH collected and calculated all data, created the tables and figures and wrote this manuscript. All authors read and approved the final manuscript.

\section{Author details}

${ }^{1}$ College of Resources and Environmental Science, Northeast Agricultural University, Harbin 150030, People's Republic of China. ${ }^{2}$ School of Life Sciences, 
Tsinghua University, Beijing 100084, People's Republic of China. ${ }^{3}$ The Shenzhen Key Laboratory of Gene and Antibody Therapy, State Key Laboratory of Health Science and Technology (prep), Center for Biotechnology \& Biomedicine and Division of Life \& Health Sciences, Graduate School at Shenzhen, Tsinghua University, Shenzhen 518055, People's Republic of China. ${ }^{4}$ Drug Safety Evaluation Center, Heilongjiang University of Chinese Medicine, Harbin 150040, People's Republic of China.

\section{Acknowledgements}

We would like to thank all authors for this manuscript.

\section{Competing interests}

The authors declare that they have no competing interests.

\section{Availability of data and materials}

Sequences used in this study were deposited to the NCBI Sequence Read Archive (Accession Number SRP067729).

\section{Ethics approval and consent to participate}

This article does not contain any studies concerned with experimentation on human. All animal experiments were approved by the Ethical Animal Care and Use Committee of Heilongjiang University of Chinese Medicine (Approval No. SCXK-2013-012) and performed in the Guidance Suggestions for the Care and Use of Laboratory Animals, formulated by the Ministry of Science and Technology of China (2006).

\section{Funding}

This work was supported by Northeast Agricultural University.

Received: 9 January 2017 Accepted: 7 February 2017

Published online: 14 February 2017

\section{References}

Adamczyk-Sowa M, Sowa P, Pierzchala K, Polaniak R, Labuz-Roszak B (2012) Antioxidative enzymes activity and malondialdehyde concentration during mitoxantrone therapy in multiple sclerosis patients. J Physiol Pharmacol 63:683-690

Bach-Knudsen KE, Hedemann MS, Laerke HN (2012) The role of carbohydrates in intestinal health of pigs. Anim Feed Sci Technol 173:41-53

Benini L, Rizzotti P, Vaona B, Sembenini C, Brocco G, Micciolo R, Chiarioni G, Pederzoli P, Vantini I, Cavallini G, Marini M, Scuro LA (1987) Elastase-1 vs trypsin, lipase and amylase serum levels in pancreatic diseases. Int J Pancreatol 2:361-370

Berry D, Schwab C, Milinovich G, Reichert J, Mahfoudh KB, Decker T, Engel M, Hai B, Hainzl E, Heider S, Kenner L, Muller M, Rauch I, Strobl B, Wagner M, Schleper C, Urich T, Loy A (2012) Phylotype-level 16S rRNA analysis reveals new bacterial indicators of health state in acute murine colitis. ISME J 6:2091-2106

Bolam DN, Sonnenburg JL (2011) Mechanistic insight into polysaccharide use within the intestinal microbiota. Gut Microbes 2:86-90

Braganza JM, Lee SH, Mccloy RF, Mcmahon MJ (2011) Chronic pancreatitis. Lancet 377:1184-1197

Brown CT, Davis-Richardson AG, Giongo A, Gano KA, Crabb DB, Mukherjee N, Casella G, Drew JC, Llonen J, Knip M, Hyoty H, Veijola R, Simell T, Simell O, Neu J, Wasserfall CH, Schatz D, Atkinson MA, Triplett EW (2011) Gut microbiome metagenomics analysis suggests a functional model for the development of autoimmunity for type 1 diabetes. PLoS ONE 6:e25782

Burcelin R, Serino M, Chabo C, Blasco-Baque V, Amar J (2011) Gut microbiota and diabetes: from pathogenesis to therapeutic perspective. Acta Diabetol 48:257-273

Daniels L, Budding AE, De-Korte N, Eck A, Bogaards JA, Stockmann HB, Consten EC, Savelkoul PH, Boermeester MA (2014) Fecal microbiome analysis as a diagnostic test for diverticulitis. Eur J Clin Microbiol Infect Dis 33:1927-1936

Fickert P, Pollheimer MJ, Beuers U, Lackner C, Hirschfield G, Housset C, Keitel V, Schramm C, Marschall HU, Karlsen TH, Melum E, Kaser A, Eksteen B, Strazzabosco M, Manns M, Trauner M (2014) Characterization of animal models for primary sclerosing cholangitis (PSC). J Hepatol 60:1290-1303
Girish BN, Rajesh G, Vaidyanathan K, Balakrishnan V (2011) Assessment of oxidative status in chronic pancreatitis and its relation with zinc status. Indian J Gastroenterol 30:84-88

Goodlad RA, Ratcliffe B, Fordham P, Wright NA (1989) Does dietary fibre stimulate intestinal epithelial cell proliferation in germ free rats? Gut 30:820-825

He C, Shan Y, Song W (2015) Targeting gut microbiota as a possible therapy for diabetes. Nutr Res 35:361-367

Hu Y, Sheng Y, Yu M, Li KK, Ren GM, Xu XH, Qu JJ (2016) Antioxidant activity of Inonotus obliquus polysaccharide and its amelioration for chronic pancreatitis in mice. Int J Biol Macromol 87:348-356

Hu Y, Shi SY, Lu L, Teng CY, Yu SM, Wang X, Yu M, Liang JS, Qu JJ (2017) Effects of selenizing modification on chara cteristics and antioxidant activities of Inonotus obliquus polysaccharide. Macromol Res 25:1-9

Inui K, Yoshino J, Miyoshi H, Yamamoto S, Kobayashi T (2013) New developments in diagnosis and non-surgical treatment of chronic pancreatitis. $J$ Gastroen Hepatol 28:108-112

Ley RE, Backhed F, Turnbaugh PJ, Lozupone CA, Knight RD, Gordon JI (2005) Obesity alters gut microbial ecology. Proc Natl Acad Sci USA 102:11070-11075

Ley RE, Hamady M, Lozupone C, Turnbaugh PJ, Ramey RR, Bircher JS, Schlegel ML, Tucker TA, Schrenzel MD, Knight R, Gordon JI (2008a) Evolution of mammals and their gut microbes. Science 320:1647-1651

Ley RE, Lozupone CA, Hamady M, Knight R, Gordon Jl (2008b) Worlds within worlds: evolution of the vertebrate gut microbiota. Nat Rev Microbiol 6:776-788

Li XM, Shi YH, Wang F, Wang HS, Le GW (2007) In vitro free radical scavenging activities and effect of synthetic oligosaccharides on antioxidant enzymes and lipid peroxidation in aged mice. J Pharmaceut Biomed 43:364-370

Li Z, Zhang Z, Xu C, Zhao J, Liu H, Fan Z, Yang F, Wright AD, Li G (2014) Bacteria and methanogens differ along the gastrointestinal tract of chinese roe deer (Capreolus pygargus). PLOS ONE 9:e114513

Li T, Long M, Gatesoupe F, Zhang Q, Li A, Gong X (2015) Comparative analysis of the intestinal bacterial communities in different species of carp by pyrosequencing. Microb Ecol 69:25-36

Lim S, Jeong J, Woo KH, Han MJ, Kim DH (2015) Lactobacillus sakei OK67 ameliorates high-fat diet-induced blood glucose intolerance and obesity in mice by inhibiting gut microbiota LPS production and inducing colon tight junction protein expression. Nutr Res 36:337-348

Ma L, Chen H, Dong P, Lu X (2013) Anti-inflammatory and anticancer activities of extracts and compounds from the mushroom Inonotus obliquus. Food Chem 139:503-508

Maslowski KM, Vieira AT, Ng A, Kranich J, Sierro F, Yu D, Schilter HC, Rolph MS, Mackay F, Artis D, Xavier RJ, Teixeira MM, Mackay CR (2009) Regulation of inflammatory responses by gut microbiota and chemoattractant receptor GPR43. Nature 461:1282-1286

Matsumura N, Ochi K, Ichimura M, Mizushima T, Harada H, Harada M (2001) Study on free radicals and pancreatic fibrosis-pancreatic fibrosis induced by repeated injections of superoxide dismutase inhibitor. Pancreas 22:53-57

Monira S, Nakamura S, Gotoh K, Izutsu K, Watanabe H, Alam NH, Endtz HP, Cravioto A, Ali Sl, Nakaya T, Horii T, lida T, Alam M (2011) Gut microbiota of healthy and malnourished children in Bangladesh. Front Microbiol 2:228

Petersen AM, Pedersen BK (2005) The anti-inflammatory effect of exercise. J Appl Physiol 98:1154-1162

Phillips P (2012) Pancreatic stellate cells and fibrosis. Pancreatic Cancer and Tumor Microenvironment, Trivandrum

Rautava S, Isolauri E (2002) The development of gut immune responses and gut microbiota: effects of probiotics in prevention and treatment of allergic disease. Curr Issues Intest Microbiol 3:15-22

Ravcheev DA, Godzik A, Osterman AL, Rodionov DA (2013) Polysaccharides utilization in human gut bacterium Bacteroides thetaiotaomicron: comparative genomics reconstruction of metabolic and regulatory networks. BMC Genom 14:873

Reilly P (2010) The effects of cereal-derived $\beta$-glucans and enzyme supplementatiation on intestinal microbiota, nutrient digestibility and mineral metabolism in pigs. Livest Sci 133:144-147

Rotruck JT, Pope AL, Ganther HE, Swanson AB, Hafeman DG, Hoekstra WG (1973) Selenium: biochemical role as a component of glutathione peroxidase. Science 179:588-590 
Schneider A, Barmada MM, Slivka A, Martin JA, Whitcomb DC (2004) Analysis of tumor necrosis factor- $\alpha$, transforming growth factor- $\beta 1$, interleukin-10, and interferon- $\gamma$ polymorphisms in patients with alcoholic chronic pancreatitis. Alcohol 32:19-24

Sehitogullari A, Aslan M, Sayir F, Kahraman A, Demir H (2014) Serum paraoxonase-1 enzyme activities and oxidative stress levels in patients with esophageal squamous cell carcinoma. Redox Rep 19:199-205

Shi LL, Li Y, Wan Y, Feng Y (2015) MDG-1, an ophiopogon polysaccharide, regulate gut microbiota in high-fat diet-induced obese C57BL/6 mice. Int J Biol Macromol 81:576-583

Silva JN, Galmiche A, Tomé JPC, Boullier A, Neves MGPMS, Silva EMP, Capiod JC, Cavaleiro JAS, Santus R, Mazière JC, Filipe P, Morlière P (2010) Chaindependent photocytotoxicity of tricationic porphyrin conjugates and related mechanisms of cell death in proliferating human skin keratinocytes. Biochem Pharmacol 80:1373-1385

Singdevsachan SK, Auroshree P, Mishra J, Baliyarsingh B, Tayung K, Thatoi H (2016) Mushroom polysaccharides as potential prebiotics with their antitumor and immunomodulating properties: a review. Bioact Carbohydr Diet Fibre 7:1-14

Siriwardena AK (2014) Reappraisal of xenobiotic-induced, oxidative stressmediated cellular injury in chronic pancreatitis: a systematic review. World J Gastroenterol 20:3033-3043

Tan CC, Ling ZX, Huang Y, Cao YD, Liu Q, Cai T, Yuan H, Liu C, Li Y, Xu K (2015) Dysbiosis of intestinal microbiota associated with inflammation involved in the progression of acute pancreatitis. Pancreas 44:868-875

Turnbaugh PJ, Ley RE, Mahowald MA, Magrini V, Mardis ER, Gordon JI (2006) An obesity-associated gut microbiome with increased capacity for energy harvest. Nature 444:1027-1031
Vanhooren V, Vandenbroucke RE, Dewaele S, Hamme EV, Haigh JJ, Hochepied T,

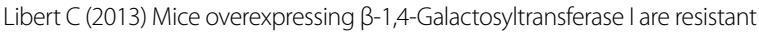
to TNF-induced inflammation and DSS-induced colitis. PLoS ONE 8:e79883

Walker AW, Sanderson JD, Churcher C, Parkes GC, Hudspith BN, Rayment N, Brostoff J, Parkhill J, Dougan G, Petrovska L (2011) High-throughput clone library analysis of the mucosa-associated microbiota reveals dysbiosis and differences between inflamed and non-inflamed regions of the intestine in inflammatory bowel disease. BMC Microbiol 11:7

Wang Y, Zhu Y, Ruan K, Wei H, Feng Y (2014) MDG-1, a polysaccharide from Ophiopogon japonicus, prevents high fat diet-induced obesity and increases energy expenditure in mice. Carbohydr Polym 114:183-189

Witt H, Apte MV, Keim V, Wilson JS (2007) Chronic pancreatitis: challenges and advances in pathogenesis, genetics, diagnosis, and therapy. Gastroenterology 132:1557-1573

Wu GD, Chen J, Hoffmann C, Bittinger K, Chen YY, Keilbaugh SA, Bewtra M, Knights D, Walters WA, Knight R, Sinha R, Gilroy E, Gupta K, Baldassano R, Nessel L, Li H, Bushman FD, Lewis JD (2011) Linking long-term dietary patterns with gut microbial enterotypes. Science 334:105-108

Xu X, Yang J, Ning Z, Zhang X (2015) Lentinula edodes-derived polysaccharide rejuvenates mice in terms of immune responses and gut microbiota. Food Funct 6:2653-2663

Yu Z, Morrison M (2004) Improved extraction of PCR-quality community DNA from digesta and fecal samples. Biotechniques 36:808-812

Zhou D, Wang W, Cheng X, Wei J, Zheng S (2015) Antioxidant therapy for patients with chronic pancreatitis: a systematic review and meta-analysis. Clin Nutr 34:627-634

\section{Submit your manuscript to a SpringerOpen ${ }^{\circ}$ journal and benefit from:}

- Convenient online submission

- Rigorous peer review

- Immediate publication on acceptance

- Open access: articles freely available online

- High visibility within the field

- Retaining the copyright to your article

Submit your next manuscript at springeropen.com 